\title{
Cortical communication and the comparison of colors
}

\section{John Mollon ${ }^{1}$ and Marina Danilova ${ }^{1,2}$}

${ }^{1}$ Department of Psychology, University of Cambridge, Downing St., Cambridge, CB2 3EB, United Kingdom

${ }^{2}$ I. P. Pavlov Institute of Physiology, Russian Academy of Sciences, nab. Makarova 6, St. Petersburg, 199034 Russia

Corresponding author: Professor J. D. Mollon,jm123@cam.ac.uk

Declaration of interest: None

\begin{abstract}
The hues or the colorimetric purities of a pair of colored targets can be compared with similar precision whether the targets are juxtaposed or fall at well-separated positions in the visual field. This is the case even if the stimuli are $10^{\circ}$ apart and fall in opposite hemifields. What could be the neural processes that underlie such comparisons? We are led to ask whether the long-range, white-matter tracts of the brain constitute a neural net (where representations are embodied in the weightings and signs of connections between the nodes of the net) or a communication network (where the same physical substrate carries different information from moment to moment).
\end{abstract}




\section{Introduction.}

In most people's lifetime, there is at least one happy period when they are refurbishing a house or apartment. A common domestic task at such times is that of matching colors using a swatch of samples of paint, fabric or carpet. Sometimes it is physically possible to superpose the sample on the surface being matched, but sometimes it is physically difficult to bring the two together. How precisely and how accurately can we match or discriminate colors? And does our discrimination deteriorate when the two stimuli lie at a distance from one another in the visual field?

The first of these questions has been intensively studied, and there exist well-honed formulae to predict the visibility of color differences for practical purposes [1]. A fundamental finding, for example, is that discrimination is finest when the discriminanda are close in chromaticity to that of the illumination to which one is adapted. But the second question - the effect of spatial separation - is only occasionally discussed [2-4]. It is on this latter issue that we concentrate here. Our psychophysical results lead us to ask what kind of network is the brain and what protocols govern transmission over the white matter tracts that connect different cortical areas. There is strong current interest in the 'connectome' [5-7], but there is relatively little recent discussion of the distinction we make here between a neural net and a communication network.

\section{The comparison of colors at a distance.}

In the case of luminance, discrimination is optimal when the discriminanda are touching one another and it deteriorates quickly as the stimuli are separated in space $[4,8]$. Yet this is not always the case for color.

[Figure 1 about here]

Figure 1 shows results from experiments in which the discriminanda varied along different axes of color space [3, 9]. Panel (a) illustrates the arrangement of stimuli. The participant is adapted to a steady white field. In order to hold eccentricity constant while the separation of the stimuli is varied, the stimulus patches are sectors of an annulus, and their midpoints lie on an imaginary circle of radius 5 degrees of visual angle. The patches are $2^{\circ}$ wide at their mid points and are brief $(<200 \mathrm{~ms})$ - too brief for a saccade from one to the other. A concurrent white marker indicates which patch is to be reported, relative to the other. In different blocks of trials, the spatial separation of the patches can vary from the case where they are touching to the case where they lie $10^{\circ}$ apart on opposite sides of the fixation point. However, although separation is held constant within a block, from trial to trial the yoked positions of the two patches fall at random places on the imaginary circle. 
An adaptive psychophysical procedure is used to measure the difference in chromaticity that allows the patches to be discriminated with a probability of $79.4 \%$ correct [3]. To ensure that the participants do actively compare the two stimuli (rather than making absolute judgments of just one of them), there is not a fixed reference chromaticity. Rather, the reference chromaticity is jittered over a range that is large relative to the threshold; and what is adjusted by the adaptive procedure is the factor by which the variable stimulus differs from the reference.

In three separate experiments we used discriminanda that varied in different directions of color space. The first two cases were for discrimination of hue: (i) The excitation of the short-wave (S) cones was varied while the ratio of excitation of the long- (L) and middle-wave (M) cones was held constant; and (ii) The excitation of the $\mathrm{S}$ cones was held constant, while the relative excitation of $L$ and $M$ cones $(L /(L+M))$ was varied.

The third data set (iii) was for variation in colorimetric purity along a line radiating from the white point and increasing in the ratio $\mathrm{L} / \mathrm{M}$, with short-wave excitation held constant. (The subjective correlate of colorimetric purity is saturation.)

The schematic MacLeod-Boynton chromaticity diagram [10] of Figure 1b shows the approximate ranges of chromaticities used in the three experiments: The vertical and horizontal lines marked (i), (ii) and (iii) correspond to the three types of variation described above.

Figure 1c shows the measured thresholds, scaled so that the value is 1.0 when the stimulus patches are touching, i.e. when their midpoints are separated by 2 degrees of visual angle. The three data sets all tell a similar story: Thresholds deteriorate very little as the stimuli are increasingly separated up to ten degrees of visual angle (even though in the latter case, they often fall into opposite hemifields.) In all three data sets, to a greater or lesser extent, discrimination is better at a small separation than when the discriminanda touch each other, a result that recalls a similar phenomenon observed in foveal vision $[2,11]$ (This 'gap effect' is to be distinguished from the 'gap effect' discussed in the literature on oculomotor saccades.)

It turns out that there are several other visual attributes (e.g. speed, spatial frequency) that similarly can be discriminated with almost equal accuracy whether they are juxtaposed or are 10 degrees apart [3]. What could be the anatomical basis for such performance?

\section{Dedicated neural comparators?}

In the case of luminance, where discrimination does deteriorate rapidly with separation, we may suppose that the observer relies on a local difference signal originating in a cell with a center-surround receptive field, a cell that draws opposed excitatory and inhibitory signals from nearby regions of the visual field. But it is difficult to imagine 
that discrimination of stimuli at any two arbitrary positions in the field is mediated by an array of dedicated comparator neurons of this kind. Such 'dipole operators', with two, separated receptive fields, have occasionally been postulated [12], and in Figure 2 we sketch a model of this kind. As an explanation of the results considered above, such a model faces a number of problems:

[Figure 2 about here]

(a) There is a combinatorial explosion in the number of dipole operators required to accommodate all possible pairings within the visual field. And separate arrays of dipole operators would be needed for (a minimum of two) dimensions of chromaticity, as well as several other dimensions, such as speed and spatial frequency. It might be objected that receptive fields become larger in more anterior parts of pre-striate cortex and so discrimination might be based on the responses of such cells. This is unlikely. If a neuron is to sub-serve one of our comparison tasks, it is not enough that it should integrate inputs for a given sensory attribute over a large area. Rather, it must signal the difference, or the ratio, of the values of the attribute in two, specific, local, and arbitrarily chosen patches of its receptive field; and it must preserve the sign of the difference. Thus far, chromatically-specific neurons with such properties have not been described in pre-striate cortex.

(b) The many dedicated connections require a significant bulk of 'wiring' - myelinated and unmyelinated axons; and the volume of wiring is known to be a critical constraint in the design of the brain [13].

(c) Thirdly, there is what we term the problem of 'junk mail'. (Unless some additional neural apparatus is postulated) the model requires each local hypercolumn in, say, V1 continuously to radiate signals about each sensory attribute to a large array of comparators - even though this broadcast information is only occasionally needed in the course of life. This is no way to run a brain, since every action potential is costly [14, 15].

(d) Perhaps the deepest problem is an extension of (a). If the result of the comparison is embodied in the activity of a dedicated comparator unit, then - for each comparator unit - a further array of dedicated connections is required to carry the information forwards to any other part of the brain that might require it for the purposes of association, decision or response. This is a general - and seldom considered - problem with the hypothesis of gnostic units or grandmother cells: If words, faces, tools, concepts and comparisons are represented centrally only by the activity of dedicated neurons - an idea implicit in much of contemporary neuroscience $[16,17]$ - then the information can be made available to other parts of the brain only by a large network of specific connections. The problem is not solved, and is possibly exacerbated, by assuming the traditional alternative to gnostic units - representation by a dedicated pattern of activity in a local ensemble of neurons: In the latter case, the distributed representation has itself to be carried forward by a projection of dedicated axons. 


\section{A communication network?}

In the general class of models considered in the preceding section, the brain is taken to be a neural net, in the sense this term is used in modern computer science. Individual nodes have a fixed response that depends on the signs and the weightings of their current connections. Although the latter are modifiable and although the 'meanings' of particular nodes may be difficult to interpret, especially in hidden layers, the response of a node is determinate for a given stimulus at a given time [18].

Neural nets may well offer a model for early stages of sensory analysis. But is the whole brain a neural net? The alternative is a communication network where the same physical substrate carries different information from moment to moment [19]. The manmade Internet offers such a model. In the case of the Internet, what are physically transmitted are ones and zeroes, but at any instant the local sequence of bits may represent a pixel, a number, an alphanumeric character or details of the source address, destination address and other control signals. Local cortical modules may resemble neural nets, but the required bandwidth of the intervening fasciculi would be vastly reduced if the fasciculi constituted a communication network. The distinction made in this essay, between two types of network, may be seen in the larger context of the classical debate between Connectionism and Symbolic AI [20].

[Figure 3 about here]

As a communication network, the white matter of the human brain is likely to be very much more parallel in architecture than is the Internet. In modern man-made networks, fiber optics allow high-speed serial transmission. In contrast, since axonal transmission is slow and since action potentials are limited in the precision of timing and in their maximal frequency, the baud rate of an individual axon cannot be high. Our illustrative model of a neuronal communication network (Fig 3), is therefore based - very loosely on the parallel architecture of a 'Small Computer System Interface' (SCSI). In many white-matter fasciculi, there is a distribution of axon diameters [21-23]. In our - strictly illustrative - model of a 'cerebral bus', we suggest that smaller fibers carry data, while the larger fibers carry control signals. Larger fibers have more rapid transmission and are energetically more costly [21]. Since they are also rarer, we tentatively suggest that they carry the simpler, but indispensable, 'hand-shaking' signals, such as 'INPUT BUFFER FULL'. A cerebral bus, of course, will differ from even a SCSI bus in two important ways: The lines - the individual axons - are not bidirectional; and the data signals, at least, may well be analogue rather than digital.

\section{The anatomical site at which colors are compared.}

Curiously, it is seldom asked where the comparison of colors is performed, i.e. where is it that the internal representations of the two signals brought together and collated? If the decision depended on an array of dedicated comparator neurons in one of the pre- 
striate areas concerned with color and if the stimuli fall in opposite hemifields, then transmission across the corpus callosum would be necessary for one of the two signals; and so some degradation of that signal might be expected, relative to the case where both signals originate within one hemisphere. In fact, providing care is taken not to bias the observer's attention, the precision of comparison of hue is the same whether the discriminanda fall in the same hemifield or in opposite ones [24].

Our own suggestion is that encoded representations of separated stimuli are independently transmitted over a 'cerebral bus' to the prefrontal cortex of one or other hemisphere; and that it is only there that the two representations are collated and compared in the form of abstract codes. The inferior occipito-frontal fasciculus would be a candidate path to carry this chromatic information forwards [25]. Diffusion tensor imaging has shown that the inferior occipito-frontal fasciculus of each hemisphere contains not only a component that originates in the ipsilateral occipital lobe but also a second component that originates in the contralateral occipital lobe and passes through the posterior corpus callosum [26]. If in fact the representations of colors are collated in prefrontal cortex, then the number of synapses is the same, and transmission distance very similar, for between-hemifield and for within-hemifield comparisons. So there is no reason to expect that one of the two signals in the inter-hemifield case might be subject to additional degradation or additional delay.

\section{Questions that are not being asked about communication within the brain.}

If, as we suggest, the long-range white-matter tracts of the brain constitute a communication network rather than a neural net, then many interesting questions immediately offer themselves. The most prominent, of course, is the format in which information is being transmitted - the long-sought 'neural code'. But there are many other questions that an engineer might ask about a man-made communication network [19]:

(i) Is information transmitted only on request, as on the Internet? This would be, in essence, the basis for selective attention. The reason that brains are likely to adopt this arrangement - we suggest - is the raw cost of broadcasting unnecessary action potentials $[14,15]$.

(ii) Is data transmission continuous or does it occur in fixed packets that might be sent by alternative routes, not only in case of damage to part of the network, but also during normal operation - as on the man-made Internet [27]?

(iii) Are addresses encoded separately from data? Are for example, the two types of information transmitted by parallel fibers?

(iv) What error checks are there to detect corrupted messages?

(v) How is the speed of transmission matched to that of the receiver? Is the currently available buffer space signaled back from the receiver to the transmitter? Do pathologies arise from failures of control mechanisms of this and the previous type? 
Some of these secondary questions might be tackled before the actual data code is understood. For example, if our hypothesis were correct, single-unit recording from the large axons of major fasciculi might reveal 'control' signals that are sparser and more stereotyped than the data signals carried by other axons. Histological examination of the terminations of the tracts might reveal structures that are analogous to the hubs of a man-made network. In this context, it is interesting that an unusual class of giant pyramidal cells have been described in layer III of the parastriate cortex at the terminations of callosal fibers [28]. It is here that the translations from one type of code to another may take place, perhaps by the mediation of dynamic synapses [29]. It will be instructive to compare the terminations of large and small fibres.

\section{Conclusions.}

Straightforward psychophysical experiments show that observers can discriminate the hues and the purities of parafoveal colors with similar precision whether the discriminanda are juxtaposed or are $10^{\circ}$ apart and fall in opposite hemifields. There is no agreed model of how (or where) this comparison is performed. Consideration of possible models leads us to ask whether the long-range tracts of the brain constitute a neural net (where representations are embodied in the nodes of the net, in the weightings and signs of connections between nodes) or a communication network, where the same physical substrate carries different information from moment to moment. We have argued in favor of the latter.

Acknowledgments. Supported by BBSRC (BB/S000623/1) and Wellcome Trust $(082378 / \mathrm{Z} / 07 / \mathrm{Z})$. We thank Dr Chie Takahashi for her kind assistance in the preparation of this paper.

\section{Figure captions}

1. a. Typical stimulus arrangement for our experiments. The participant fixates a central white dot, and the target patches - the 'discriminanda' - fall on an imaginary circle of diameter $10^{\circ}$. A white bar marker, concurrent with the discriminanda, indicates which patch is to be judged as, say, 'more saturated'. $b$. A local region of a MacLeod-Boynton chromaticity diagram [10]. The ordinates of this diagram are thought to correspond to two physiological signals - cone ratios - extracted at an early stage of the visual system [30]. 'G' and 'R' indicate the green and red phosphors of the display, and the dotted line shows part of the spectrum locus. The vertical and horizontal lines marked (i) to (iii) indicate the approximate ranges of chromaticities tested in the three experiments. The present diagram is strictly schematic, since different white points were used in the different experiments (Equal-energy white vs Illuminant D65) and since chromaticity was jittered within ranges that were chosen on the basis of preliminary measurements for each participant. $c$. The separate panels show the relationship between normalized threshold and the spatial separation of the two 
patches. The left and middle panels show results for hue discrimination along lines parallel to the vertical (i) and horizontal (ii) ordinates of the MacLeod-Boynton diagram; and the right-hand panel shows results for purity discrimination along an axis where the ratio of long-wave to middle-wave cone excitation is varied (iii). The data are averaged across participants and the error bars represent standard errors based on betweensubject variance. To allow comparison of the different axes, the thresholds in each case are scaled relative to the threshold when the edges of the discriminanda are touching.

2. A model in which discriminations are performed by dedicated 'comparator units'. At the lower level, representing early visual cortex, chromatically opponent cells draw their inputs from local areas of the visual field. At the second level, there is a dedicated comparator unit for each possible pairing of cells at the first stage. Each comparator unit draws opposed excitatory and inhibitory inputs from the paired lower-order cells. So for $n$ cells at the lower level, $(n(n-1)) / 2$ comparators are required (and the matrix of comparators would need to be doubled if only positive ratios are signaled by each comparator - as is usually assumed to be the case for midget ganglion cells in the retina). A further array of dedicated projections is then needed to convey the results of the comparison forwards to other parts of the cortex that might need the information.

3. A cross-section of part of a 'cerebral bus'. This might be, say, part of the inferior fronto-occipital fasciculus, delivering information from different parts of the visual field to the prefrontal cortex. In all white-matter tracts there is range of axon diameters [23] and in this illustrative example (of what is a large generic class of models) we assign Controlsignals to the minority types of large axons, which have faster transmission, and we assign Data signals to the many smaller axons. Control signals sub-serve the 'handshaking' between transmitter and receiver that has been proved a necessary feature of almost all man-made communication networks [19]. If in fact the larger axons do carry control signals, then a testable hypothesis might be that their signals are more stereotyped than those of the smaller, data-carrying axons. 


\section{References}

1 Fairchild, M.D. (2015) Color models and systems. In Handbook of Color Psychology (Elliot, A.J., et al., eds), pp. 9-26, Cambridge University Press

* An authoritative introduction to the concepts of color science and the properties of color spaces.

2 Saeedi, H. and Kandi, S.J. (2019) How anisotropy of CIELAB color space affects the separation effect: an experimental study. Journal of the Optial Society of America 36, 5160

3 Danilova, M.V. and Mollon, J.D. (2018) Cerebral iconics: how are visual stimuli represented centrally in the human brain? Journal of Optical Technology 85, 515-520

* A recent paper that gives more experimental detail of how our psychophysical data are obtained.

4 Traub, A.C. and Balinkin, I. (1961) Proximity factor in the Judd color difference formula. Journal of the Optical Society of America 51, 755-760

5 Bassett, D. and Sporns, O. (2017) Network neuroscience. Nature Neurosci 20, 353-364

* This paper, and the following two, offer accessible introductions to neuroscientic applications of network theory.

6 Navlakha, S., et al. (2018) Network Design and the Brain. Trends Cogn Sci 22, 64-78

7 Bassett, D.S., et al. (2018) On the nature and use of models in network neuroscience.

Nature reviews $19,566-578$

8 Le Grand, Y. (1933) Sur la précision en photométrie visuelle. Revue d'optique

théoretique et instrumentale 12, 145-159

9 Danilova, M.V. and Mollon, J.D. (2006) The comparison of spatially separated colours.

Vision Research 46, 823-836

10 MacLeod, D.I.A. and Boynton, R.M. (1979) Chromaticity diagram showing cone excitation by stimuli of equal luminance. Journal of the Optical Society of America 69, 1183-1186

11 Eskew, R.T. (1989) The gap effect revisited: Slow changes in chromatic sensitivity as affected by luminance and chromatic borders. Vision Research 29, 717-729

12 Balas, B.J. and Sinha, P. (2003) Dissociated dipoles: Image representation via nonlocal comparisons. MIT

13 Chklovskii, D.B. and Koulakov, A.A. (2004) Maps in the brain: what can we learn from them? Annu Rev Neurosci 27, 369-392

14 Sterling, P. and Laughlin, S.B. (2015) Principles of Neural Design. MIT

** A distinguished and readable essay on how the morphology and the biophysics of neurons are constrained by energy limitations and by the type of information carried. 15 Lennie, P. (2003) The cost of cortical computation. Current Biology 13, 493-497 16 Coltheart, M. (2017) Grandmother cells and the distinction between local and distributed representation. Language, Cognition and Neuroscience 32, 350-358 17 Thomas, E. and French, R. (2017) Grandmother cells: much ado about nothing. Language, Cognition and Neuroscience 32, 342-349

18 Yamins, D.L. and DiCarlo, J.J. (2016) Using goal-driven deep learning models to understand sensory cortex. Nat Neurosci19, 356-365

19 Tanenbaum, A.S. and Wetherall, D.J. (2014) Computer Networks. Pearson

** This is a standard text on communication networks (as opposed to neural nets). It has centrally influenced the proposals put forward by the present authors. 
20 Boden, M.A. (2006) Mind as Machine: A History of Cognitive Science. Oxford University Press

21 Perge, J.A., et al. (2012) Why do axons differ in caliber? J Neurosci 32, 626-638

22 Aboitiz, F., et al. (1992) Fiber composition of the human corpus callosum. Brain Res $598,143-153$

23 Liewald, D., et al. (2014) Distribution of axon diameters in cortical white matter: an electron-microscopic study on three human brains and a macaque. Biol Cybern 108, 541-557

24 Danilova, M.V. and Mollon, J.D. (2009) The symmetry of visual fields in chromatic discrimination. Brain and Cognition 69, 39-46

25 Takemura, H., et al. (2017) Occipital White Matter Tracts in Human and Macaque. Cereb Cortex 27, 3346-3359

26 Sherbondy, A., et al. (2005) Exploring connectivity of the brain's white matter with dynamic queries. IEEE Transactions on Visualization and Computer Graphics 11, 419430

27 Graham, D.J. (2014) Routing in the brain. Front Comput Neurosc 8

28 Braak, E. and Braak, H. (1985) On layer III pyramidal cells in the parastriate borderzone of man. J Hirnforsch 26, 117-125

29 Liaw, J.S. and Berger, T.W. (1996) Dynamic synapse: a new concept of neural representation and computation. Hippocampus 6, 591-600

30 Viénot, F. (2016) Cone fundamentals: A model for the future of colorimetry. Lighting Research and Technology 48, 5-13

* A tutorial guide to the construction of chromaticity diagrams of the MacLeod-Boynton type. 

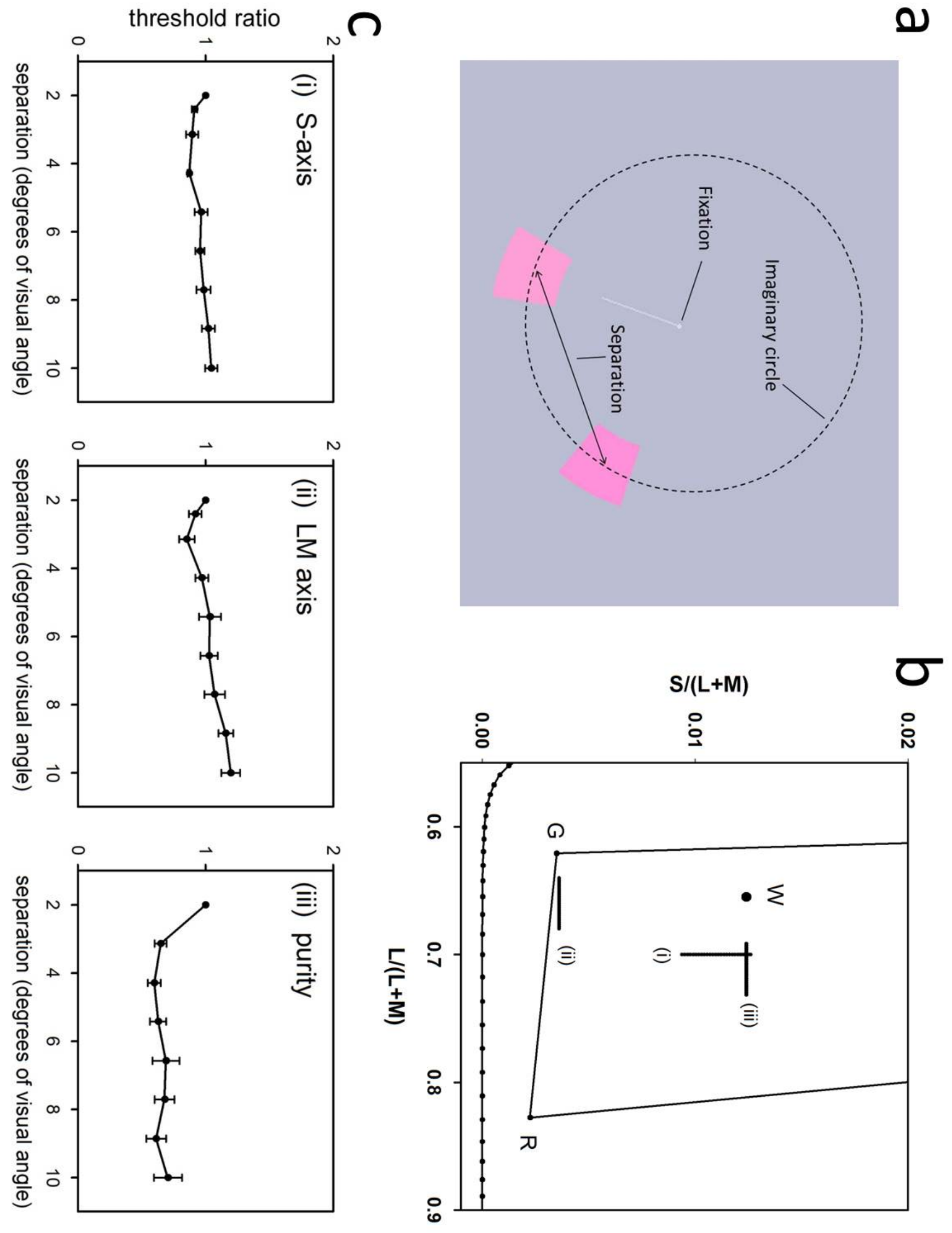


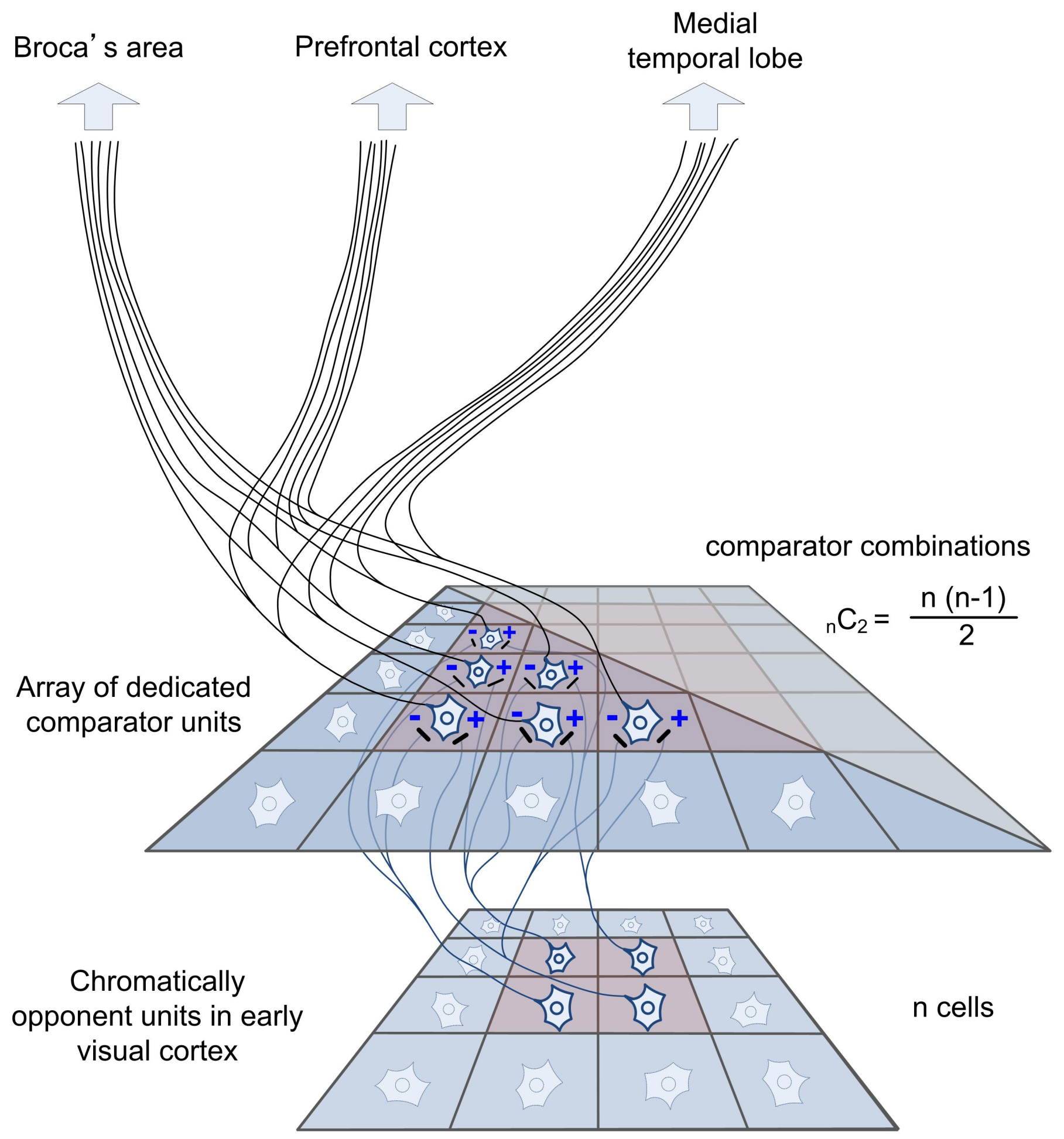



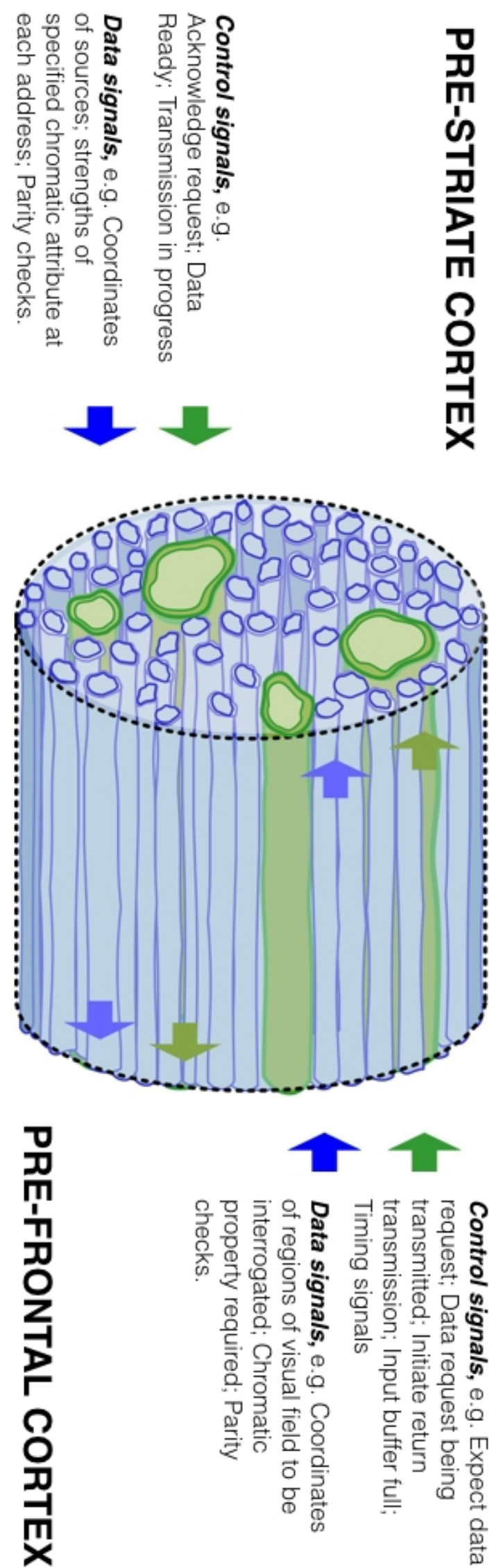
Declaration of interests: None 